Results Patients who have undergone conchal cartilage cap ossiculoplasty for stapes suprastructure showed good hearing improvement. Postoperative follow-up of ABG for a period of 1 year in 23 patients demonstrated significant audiometrical improvement. There were no ABG of more than $30 \mathrm{~dB}$ and mean $\mathrm{ABG}$ of $20 \mathrm{~dB}$ was achieved for most of the cases.

Conclusion The technique of conchal cartilage cap for stapes suprastructure ossiculoplasty is a safe, simple, reliable, and effective procedure with an easy learning curve. Hearing results are satisfactory and comparable to other commonly applied techniques and materials. Compared with synthetic prostheses, conchal cartilage ossiculoplasty also offers better biocompatibility at no additional expenditure.

Clinical Significance Development of an undemanding, consistent and easily performed technique of ossiculoplasty using autologous cartilage at no extra cost provides another option in the armamentarium of an otologist for good hearing outcomes in mastoid surgery.

\section{A0022: Cochlear Implants in Auditory Neuropathy Spectrum Disorder: Role of Electrically Evoked Auditory Brainstem Responses and Serial Neural Response Telemetry \\ Aanchal Aggarwal}

Objective To evaluate the utility of neural response telemetry (NRT) and electrically evoked auditory brainstem response (EABR) in patients with auditory neuropathy spectrum disorder (ANSD)

Methods Four patients with ANSD who underwent cochlear implantation and usage for more than 1 year were studied. All the four patients underwent preoperative transtympanic EABR (TT-EABR), intraoperative neural response telemetry (NRT), postoperative NRT at 3 months, 6 months, and 1-year intervals after switch-on and outpatient $\mathrm{CI}$ (cochlear implant) EABR testing at 1-year post switch-on.

Results and Discussion The authors propose a new practical classification of understanding the neural responses in relation to TT-EABR wave-form morphology and latencies. NRT and/or EABR measurements showed improvements in all the four patients. Three out of the four patients had NRTs on three or more electrodes and all the four patients had EABRs at 1 year of implant use. In addition, it was apparent that the children with better wave-form morphology on TTEABR preoperatively had better category of auditory performance (CAP) and speech intelligibility ratings (SIRs) scores at 1 year of implant use.

Conclusions Improvements in EABR and NRT over time with $\mathrm{CI}$ use indicates that electrical stimulation is a favorable scheme of auditory stimulation in ANSD patients. This also provides an objective way to monitor changes/progress in the auditory pathways after cochlear implantation.

\section{A0023: Diagnosis and Treatment of Menière's Disease:} Our Experience

Akash A. R.

Introduction Menière's disease is a clinical syndrome that consists of episodes of spontaneous incapacitating vertigo usually associated with unilateral fluctuating sensorineural hearing loss (SNHL), tinnitus, and aural fullness. Diagnosis is by clinically and audiovestibular tests including video nystagmography (VNG).

Aim (1) This study was done to determine whether video nystagmography is a valid diagnostic tool for diagnosing Menière's disease.

(2) To study the therapeutical effect of betahistine in Menière's disease and compare with video nystagmography.

Methodology Patients, presenting with vertigo, tinnitus, and fluctuating hearing loss, who come under the criteria for Menière's disease in the ENT OPD were selected. A detailed history and thorough clinical examination were done followed by PTA and video nystagmography. Those who are diagnosed with Menière's disease were treated with betahistine $48 \mathrm{mg}$ for 2 weeks, and were followed-up for 2 and 6 weeks.

Results Thirty-five patients have been studied, all of them showed nystagmus toward the affected ear on post high-frequency head shake, hypofunction on caloric test in affected side. Twenty patients were correlated with glycerol test and PTA showing SNHL on affected side. On follow-up, 15 patients showed improvement clinically and in VNG. These tests were done on follow-up at 2 and 6 weeks to look for improvement.

Conclusion VNG is an excellent tool for diagnosing Menière's disease and to compare the therapeutical improvement or efficacy of treatment given.

Clinical Significance Menière's disease can be easily diagnosed in OPD setup with VNG, it helps in accurate and early treatment of the patient.

A0024: Comparative Study of Palisade Cartilage Tympanoplasty with Temporalis Fascia Tympanoplasty in CSOM with Subtotal Perforations

Sathish Kumar K. N., ${ }^{1}$ M. K. Veenapani, ${ }^{1}$ Akash Aradhya S., ${ }^{1}$ Swathi V. M. ${ }^{1}$

${ }^{1}$ Mysore Medical College, Mysuru, Karnataka, India

Objective To assess and compare the graft acceptance rates and audiological outcomes of palisade cartilage tympanoplasty with those of temporalis fascia tympanoplasty in CSOM with subtotal perforation.

Materials and Methods A prospective study containing 50 patients with the diagnosis of CSOM with subtotal perforations attending the department of ENT, K. R. Hospital between January 2017 and December 2017. Patients were divided into two groups with equal number of patients in 